Paper presented at Innovative Smart Grid Technologies Conference (ISGT), 2015 IEEE Power \& Energy Society Washington DC, 18-20 Feb 2015. DOI:10.1109/ISGT.2015.7131814

\title{
Simulation Approach to Reliability Analysis of WAMPAC System
}

\author{
Yan Zhang, Mats Larsson \\ Department of Automation \\ ABB Corporate Research \\ Baden, Switzerland \\ \{yan.zhang, mats.larsson\}@ch.abb.com
}

\author{
Bikash Pal, Nina F. Thornhill \\ Department of Electrical, Chemical Engineering \\ Imperial College \\ London, United Kingdom \\ \{b.pal, n.hornhill\}@imperial.ac.uk
}

\begin{abstract}
Wide are monitoring, protection and control (WAMPAC) plays a critical role in smart grid development. Since WAMPAC frequently has the tasks of executing control and protection actions necessary for secure operation of power systems, its reliability is essential. This paper proposes a novel approach to the reliability analysis of WAMPAC systems. WAMPAC system functions are first divided into four subsystems: the measured inputs, the communication, the actuator and the analytic execution subsystems. The reliability indices of the subsystems are computed then using Monte Carlo approach. A sensitivity analysis is also described to illustrate the influence of different components on the system reliability.
\end{abstract}

Index Terms-Reliability, wide area networks, wide area measurements, Monte Carlo methods

\section{INTRODUCTION}

A WAMPAC system includes a wide range of power system applications in the area of monitoring, protection and control. It operates over a wide area communication network, connecting with various number of sensors and actuators [1]. Reliability analysis is important both for the design and operation of such a system. A WAMPAC system may fail when any of its subsystem such as communication or actuators fail and may consequently lead to serious and costly consequences. Therefore, it is important to evaluate the reliability of WAMPAC system quantitatively.

Reliability in power systems has been extensively studied. There is a significant number of reliability assessment methodologies for power system applications [2-4]. The methods employed are mostly based on either analytical or simulation approaches. Examples of analytical approaches are Reliability Block Diagram, Event Tree, Fault Tree and Marko Modelling methods [5]. Simulation approach are often based on Monte Carlo or simulations with stochastic distribution methods. Reference [6] provides a detailed reliability assessment of operational functions of a power system control centre. Reference [7] evaluates the reliability of a SCADA system considering its performance requirements. Both of these approaches are using the method of fault tree analysis.

The financial support from the Marie Curie FP7-IAPP project "Using real-time measurements for monitoring and management of power transmission dynamics for the Smart Grid - REAL-SMART", Contract No: PIAP-GA-2009-251304 is gratefully acknowledged.
There is little work on the assessment of WAMPAC system reliability. Discussions can be found only focusing over wide area communications [8]. In principle, the same reliability analysis approach applied to SCADA or control centre can be adopted for WAMPAC system. It is however infeasible to model a large number of real system components and their dependent reliability characteristics by analytical approaches. Thus, they are not suitable for WAMPAC system reliability analysis. Especially when state estimation methods are employed, it is impossible to put the system into analytical blocks. Also analytical approach have difficulties to consider communication delays.

To overcome the limitations of the analytical methods, this paper presents a novel and alternative approach to reliability analysis of WAMPAC system. WAMPAC system functions are first divided into four subsystems: measurement input, the wide area communication, the actuator and the analytic execution subsystems. The reliability indices of the entire system are then computed using simulation (a Monte Carlo approach). A sensitivity analysis illustrates also the process of evaluation the importance of different system components.

\section{WAMPAC SYSTEM RELIABILITY}

WAMPAC systems are often based on the new data acquisition technology of phasor measurement units (PMUs). These PMUs are placed at selected primary substations to obtain measurements by sensors and to execute control actions through actuators. The PMUs are able to report their measurement values upwards to a hierarchy of substations for phasor data concentrators (PDCs), then to network control centres through available communication networks [9]. A centralized control unit, often located in a control centre, make decisions and sends control signals back to the actuators through remote terminal units (RTUs) or PMUs.

Figure 1 shows a simplified sketch of a WAMPAC system. A WAMPAC operation presupposes a wide area communication network, which may have different structures depending the communication media and security requirements [10]. In practice, the physical communication network normally consists of a broadband backbone. 


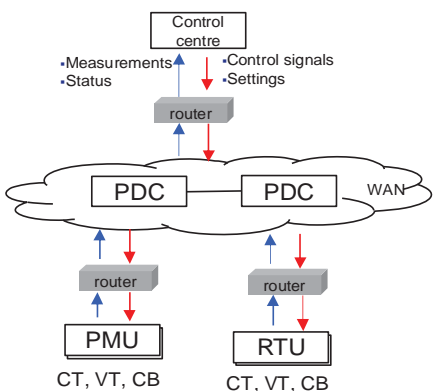

Figure 1. Sketch of a WAMPAC application

\section{A. Failure analysis of WAMPAC system}

A WAMPAC system includes a wide range of power system applications in the area of monitoring, protection and control. A WAMPAC system may fail when the operator is unable to retrieve data from or issue controls to associated buses/devices. The cause and effect diagram also known as fishbone diagram is used here to illustrate the possible causes of a WAMPAC system failure, as shown in Figure 2. A WAMPAC system can be divided into four subsystems: the measurement input, the wide area communication, the actuator and the analytic execution subsystems. Each subsystem is a combination of various of system components, such as router, circuit breakers and PMUs.

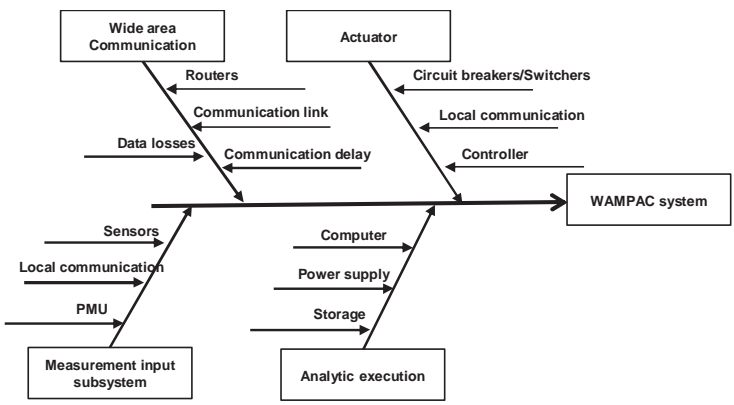

Figure 2. Failure causes of a WAMPAC system

For example, a measurement input subsystem consists measurement instruments such as CT and VT, a local communication network and PMU devices. The functional structure of this subsystem can be described as Figure 3.

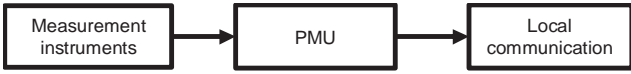

Figure 3. System structure of a measurement input subsystem

The reliability of such a measurement input subsystem is then obtained by combined reliability of the component (measurement instruments, PMU, local communication) in a series configuration as described as:

$$
R\left(S_{i}\right)=R\left(p_{1}\right) \cdot R\left(p_{2}\right) \cdot R\left(p_{3}\right)
$$

where $R\left(S_{i}\right)$ denotes the reliability of measurement input subsystem $S_{i}, R\left(p_{1}\right), R\left(p_{2}\right), R\left(p_{3}\right)$ denote the reliability of component 1,2, 3 (measurement instruments, PMU and local communication) respectively. If the reliability of all components of the subsystem is known to be same as 0.99 , the reliability of the subsystem is $0.99 \times 0.99 \times 0.99=0.9703$.

\section{B. Wide area communication subsystem}

The subsystem actuator, analytic execution subsystem can be also modulated as the example as measurement input subsystem. The wide area communication subsystems are however more complicated. They consist of a broadband backbone and of user access equipment such as modems, routers or multiplexers. The backbone network uses mostly fiber optic cables, but may be supplemented by copper wire and microwave radio sections, and - for WANs operated by power transmission utilities - also by power line carrier communication (PLC) links. WAMPAC systems normally include one or several PDCs that collect and sort the data from the PMUs. The reliability of WAN might be different at different user access points due to various numbers of communication hubs the measurements at the access points must go through to reach the control centre. The reliability of such a subsystem then must be accessed case by case for each measurement input, actuator and analytic execution subsystem.

\section{Importance of WAMPAC subsystem and components}

It is obvious that some components (such as routers, PDCs) in a WAMPAC system are more important for the system reliability than other components. A component in series with the rest of the system will, for example, be at least as important as any other component in the system. There are various measures defined for the importance of components [11], such as Birnbaum's measure, the improvement potential measure. Birnbaum's measure can be described as:

$$
I\left(p_{i}\right)=R\left(1_{p i}\right)-R\left(0_{p i}\right)
$$

where $I(i)$ is the importance measure of component $p_{i}$ $R\left(1_{p i}\right)$ is the system reliability when the reliability of component $p_{i}=1, R\left(0_{p i}\right)$ is the system reliability when the reliability of component $p_{i}=0$.

The importance of components not only depends on its location and function, but also on the WAMPAC applications. A component may be very important for a wide area control application but may have little, or no importance for a wide are monitoring application.

\section{WAMPAC system reliability assessment appraoch}

The computational procedure for reliability analysis of a WAMPAC system is defined as follows:

1) Evaluate the reliability of subsystems: measurement input, actuator and analytic execution using the method introduced in section B.

2) Evaluate the data requirements for examining WAMPAC application by defining the inputs and outputs of the application.

3) Gather all available measurement input subsystems.

4) Build a graph by assigning all available measurement input subsystems and actuator subsystems as the leaf nodes, anlytic excution subsystems as the root node, the wide area communication subsystem components such as routers as the connecting nodes. The wide 
area communication links are as the edges in the graph.

5) Perform Monte Carlo simulation as follows:

\begin{tabular}{|l|}
\hline Loop 1:N (Monte Carlo simulation) \\
Generate status of all the nodes and link \\
in the graph based on reliability; \\
Find all shortest path trees from \\
available leaf nodes to root nodes; \\
if a node is disconnected \\
related node is disabled; \\
end \\
if latency > threshold \\
related node is disabled; \\
end \\
Observability analysis if it exists; \\
if system is not observable or required \\
actuator nodes is not available \\
record as system failure $N_{f}$ \\
end
\end{tabular}

6) Calculate the reliability by $R=N_{f} /_{N}$, where $R$ is the system reliabiliyt, $N$ is the Monte Carlo simuation number, and $N_{f}$ is the recorded system failure number.

The graph theory is applied in the simulation. The communication delays due to communication lines are taken into account as the weights of edges. The communication delays due to routers and PDCs processing are considered as the weights of connecting nodes. The computation delays at the analytic execution subsystems are considered as the weights of the root nodes.

State estimation can give an estimation of the system state variables of the system based on existing measurements. A state estimation may be used but it is not necessary. One of the advantages of employment of state estimation is the loss of some measurements may not halt the WAMPAC application. One of the disadvantages is the increased computation delays. The reliability of state estimation is also a concern.

\section{SimUlATION AND DISCUSSION}

An example network equipped with a loading shedding scheme implemented based on WAMPAC demonstrates the proposed reliability analysis approach.

\section{A. Test network and assumptions}

The example network considered is shown in Figure 4. A distinct generation centre can be found in the area north of Cut 1 , which contain three major generators and some shunt compensation but only a few minor loads. Between Cuts 1 and 2 is an area with no generation equipment and only a few minor loads. South of Cut 2 is an area with predominantly the characteristics of load.

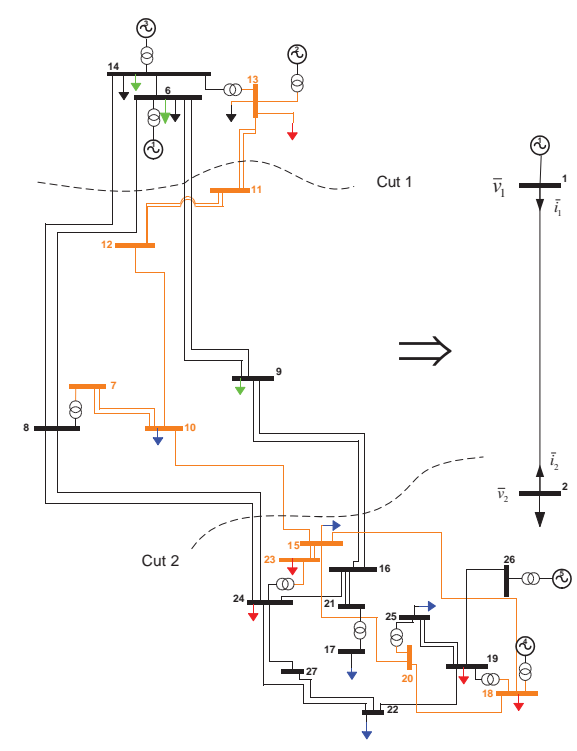

Figure 4. Single line diagram of the used test system

In the example a voltage stability protection can be achieved by load shedding controls at buses 17, 22 and 25 . The complete engineered voltage stability management logic can be seen in Figure 5.

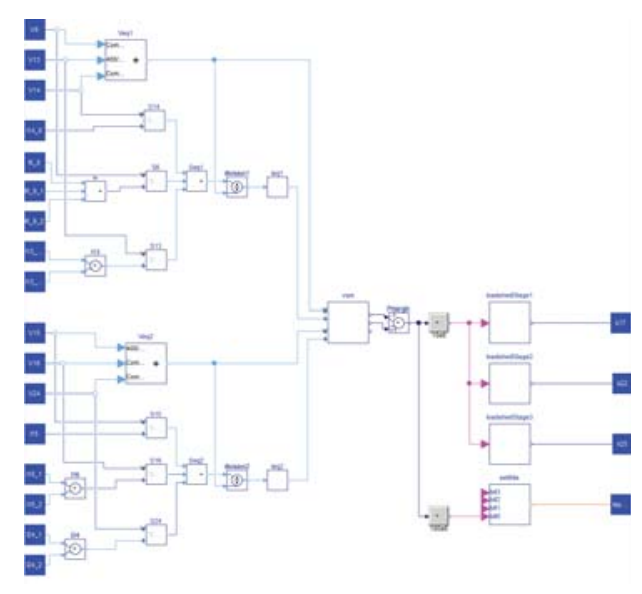

Figure 5. Load shedding logic used in the case study

It is assumed that the application is PMU-based. In total, 21 PMUs and 3 RTUs are placed in the system as shown in TABLE I. The voltage stability evaluation and load shedding scheme logic are executed at the control centre. It must have voltage and current status of substation 6, 13, 14, 15, 16, 24 through PMUs or based on state estimation and send control signals to substation 17, 22 and 25 through RTUs.

TABLE I. PMU AND RT LOCATION IN THE TEST NETWORK

\begin{tabular}{|c|c|c|}
\hline & PMUs & RTUs \\
\hline Substation number & $1,2,3,4,5$, & $17,22,25$ \\
& $6,13,14,15,16,24$ & \\
\hline
\end{tabular}


The reliability value of system components are presented as failure rate. The numbers used for the simulations are listed in TABLE II. The reliability value is calculated by (constant failure rate is assumed):

$$
R\left(p_{i}, t\right)=e^{-\lambda t}
$$

where $R\left(p_{i}, t\right)$ is the reliability of component $p_{i}$ at time $t, \lambda$ is the constant fault rate. Here it is also assumed that the reliability does not change with $t$. So $t=1$.

The latency requirements vary with applications. Depending on the control function, cycle response times for wide area control range from minutes down to $10 \mathrm{~ms}$ [12]. In the case studies, it is assumed that the latency limit for the load shedding scheme is $1 \mathrm{~s}$.

TABLE II. RELIABILITY AND LATENCY VALUE APPLIED IN TEST

\begin{tabular}{|l|l|l|l|}
\hline & failure/year & reliability & Latency \\
\hline Communication line & $500 \times 10-6$ & 0.9995 & $1 \mathrm{~ms}$ \\
\hline Routing nodes & $500 \times 10-6$ & 0.9995 & $1 \mathrm{~ms}$ \\
\hline Routers & $500 \times 10-6$ & 0.9995 & $1 \mathrm{~ms}$ \\
\hline PMUs, RTUs & $500 \times 10-6$ & 0.9995 & $20 \mathrm{~ms}$ \\
\hline Circuit breakers & $500 \times 10-6$ & 0.9995 & $20 \mathrm{~ms}$ \\
\hline Sensors & $500 \times 10-6$ & 0.9995 & $20 \mathrm{~ms}$ \\
\hline Local communication & $500 \times 10-6$ & 0.9995 & $1 \mathrm{~ms}$ \\
\hline Control centre & $500 \times 10-6$ & 0.9995 & $20 \mathrm{~ms}$ \\
\hline
\end{tabular}

\section{B. Simulations}

The communication infrastructure is simplified in the case studies. The PMUs are assumed to reside in substations, connected to a local area network, which is connected via a substation router to a WAN. The PMU based application load shedding resides at a central location connected to WAN through local communication.

In order to evaluate the reliability for the load shedding scheme, a number of cases are simulated. Two communication structures are evaluated:

- Case A: ring connection. A skeletal network structure is shown in Figure 6. The octagon shape nodes $R_{i}$ $(i=1,2,3,4)$ represent 4 routing nodes on the backbone network. The backbone network has a ring topology in this case. The routing nodes may consist of various numbers of high-performance routers. The circle shape nodes $S_{i}(i=1,2, \ldots, 24)$ represents the PMU-equipped substation. The circle shape CE represents the control centre. Substations 1,2,3,6, 13 and 14 are connected to R2. Substations 17, 22 and 25 are connected to R4. Substations 4, 5, 15, 16 and 24 are connected to R2. CE is connected to R1.

- Case B: meshed connection. A skeletal network structure is shown in Figure 7. The backbone network in this case is completely meshed. Others are same as in Case A.
- $\quad$ Case C: hierarchical connection. A skeletal network structure is shown in Figure 8. Here the PDCs are applied to gather all the data. The substations are connected to PDCs through WAN. CE is connected to PDCs also through WAN.

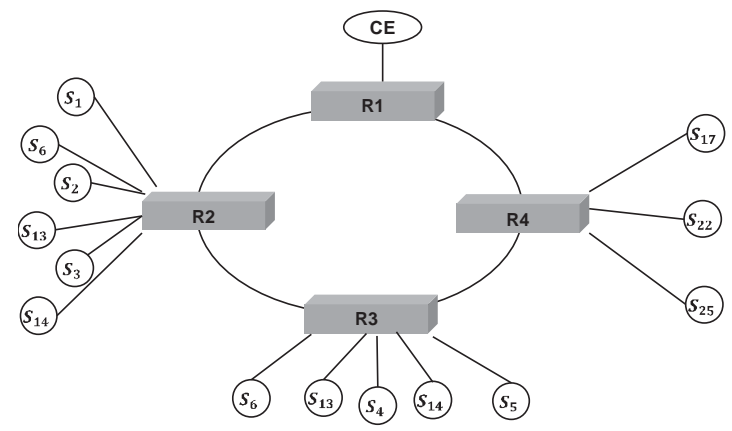

Figure 6. Ring connection of backbone network skeletal structure

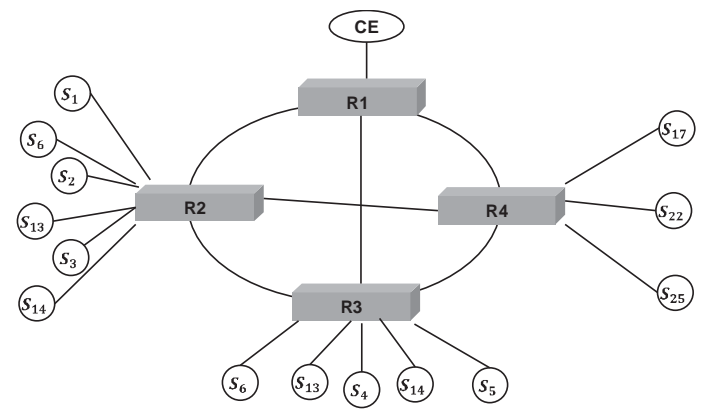

Figure 7. Meshed connection of backbone network skeletal structure

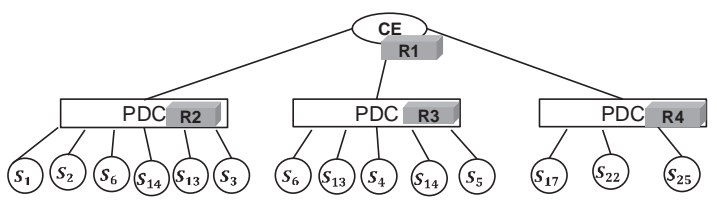

Figure 8. Hierachical connection skeletal structure

The substations are first examined and the reliability value of the substations of measurements input, actuator and analytic execution are calculated using the method introduced in section II.A. The results are listed in Table III.

TABLE III. SIMULATION RESULT -1 SYSTEM RELIABILITY

\begin{tabular}{|l|l|l|}
\hline substations & $\begin{array}{l}\text { types of } \\
\text { subsystems }\end{array}$ & reliability \\
\hline $\mathrm{s}_{1,2,3,6,13,14}$ & $\begin{array}{l}\text { measurement } \\
\text { input }\end{array}$ & 0.9980 \\
\hline $\mathrm{s}_{4,5,3,15,16,24}$ & $\begin{array}{l}\text { measurement } \\
\text { input }\end{array}$ & 0.9980 \\
\hline $\mathrm{s}_{17,22,25}$ & actuator & 0.9980 \\
\hline
\end{tabular}

The three cases are simulated, first without state estimation, then with state estimation. Some of test results are presented in TABLE IV. It can be seen that the hierarchical connection has the worst reliability. It is true because all the measurements connected to the PDC will lost if the communication link between $\mathrm{PDC}$ and $\mathrm{CE}$ is no more available. 
TABLE IV. SIMULATION RESULT -1 SYSTEM RELIABILITY

\begin{tabular}{|l|l|l|l|}
\hline & \multicolumn{1}{|c|}{ Case A } & \multicolumn{1}{|c|}{ Case B } & \multicolumn{1}{c|}{ Case C } \\
\hline $\begin{array}{l}\text { non state } \\
\text { estimation }\end{array}$ & 0.986 & 0.99 & 0.95 \\
\hline State estimation & 0.994 & 0.996 & 0.98 \\
\hline
\end{tabular}

Simulations also carried out to illustrate the importance of system components. The measure of importance is calculated based on the Birnbaum's measure as introduced in Section II.C. The results are shown in TABLE V.

TABLE V. SIMULATION RESULT 2 - SYSTEM RELIABILITY IMPROVEMENT

\begin{tabular}{|c|c|c|c|}
\hline \multicolumn{4}{|c|}{ case A } \\
\hline \multicolumn{2}{|c|}{ components } & $\mathrm{R} 1$ & S1 \\
\hline \multirow{3}{*}{$\begin{array}{l}\text { no state } \\
\text { estimation }\end{array}$} & Reliability $=1$ & 0.986 & 0.986 \\
\hline & Reliability $=0$ & 0 & 0.98 \\
\hline & improvement & 0.986 & 0.006 \\
\hline \multicolumn{4}{|c|}{ case B } \\
\hline & & $\mathrm{R} 1$ & $\mathrm{~S} 1$ \\
\hline \multirow{3}{*}{$\begin{array}{l}\text { no state } \\
\text { estimation }\end{array}$} & Reliability $=1$ & 0.990 & 0.990 \\
\hline & Reliability $=0$ & 0 & 0.991 \\
\hline & improvement & 0.99 & 0.001 \\
\hline \multicolumn{4}{|c|}{ case $\mathrm{C}$} \\
\hline & & $\mathrm{R} 1$ & $\mathrm{~S} 1$ \\
\hline \multirow{3}{*}{$\begin{array}{l}\text { no state } \\
\text { estimation }\end{array}$} & Reliability $=1$ & 0.95 & 0.956 \\
\hline & Reliability $=0$ & 0 & 0.947 \\
\hline & improvement & 0.95 & 0.009 \\
\hline
\end{tabular}

\section{CONCLUSIONS}

Generally analytical methods are applied to reliability analysis for SCADA or control centre applications. It is however difficult to apply analytical approach to the reliability analysis for WAMPAC system applications due to the large number of system components involved and the complexity of wide area communication network structure. In this paper, a simulation based reliability analysis approach is presented. This approach allows handling the complexity of the WAMPAC system applications.

The proposed approach is based on Monte Carlo simulations. A WAMPAC system is first divided into four types of subsystems: measurement input, actuator, wide area communication and analytic execution subsystems. Then subsystems are mapped into graph nodes and edges. The shortest path tree search is employed to find all the available measurements and control signals communication paths with minimal delay in the system. The latency caused due to communication or computation are modelled as the weights of graph nodes and edges. The influence of state estimation on reliability of the WAMPAC system can also be analysed by a observability analysis in this approach.

Several case studies are performed to demonstrate the potential of the proposed approach on WAMPAC system evaluation, design and planning.

\section{ACKNOWLEDGMENT}

The authors gratefully acknowledge the contributions of Thanikesavan Sivanthi and Dacfey Dzung.

\section{REFERENCES}

[1] Terzija, Vladimir, "Wide Area Monitoring Protection and Control WAMPAC," Information and Communication Technology in Electrical Sciences (ICTES 2007), 2007. ICTES. IET-UK International Conference on , vol., no., pp.I-1,I-1, 20-22 Dec. 2007

[2] Bulk Power System Reliability Assessment-Why And How? Part I and Part II: Why?," Power Apparatus and Systems, IEEE Transactions on , vol.PAS-101, no.9, pp.3439,3445, Sept. 1982

[3] Richard E. Brown, 'Electric Power Distribution Reliability, Second Edition', CRC Press, September 9, 2008

[4] J. Endrenyi and W. H. Wellssow, "Power system reliability in terms of the system's operating states," in Power Tech Proceedings, 2001 IEEE Porto, 2001, p. 6 pp. vol.2.

[5] Roy Billinton and R. N. Allan, reliability evaluation of engineering systems: Concepts and Techniques, 2nd ed.: Plenum Press, 1992.

[6] Wang, L.; Gelberger, P.P.; Ramani, N., "Reliability assessment of the operational functions of a power system control center," Probabilistic Methods Applied to Electric Power Systems, 1991., Third International Conference on , vol., no., pp.229,234, 3-5 Jul 1991

[7] Chun-Lien Su; Ya-Chin Chang, "A SCADA system reliability evaluation considering performance requirement," Power System Technology, 2004. PowerCon 2004. 2004 International Conference on, vol.1, no., pp.574,579 Vol.1, 21-24 Nov. 2004

[8] Kun Zhu; Chenine, M.; Nordstrom, L., "ICT Architecture Impact on Wide Area Monitoring and Control Systems' Reliability," Power Delivery, IEEE Transactions on, vol.26, no.4, pp.2801,2808, Oct. 2011

[9] Salehi, V.; Mazloomzadeh, A; Fernandez, J.F.; Mohammed, O.A, "Realtime power system analysis and security monitoring by WAMPAC systems," Innovative Smart Grid Technologies (ISGT), 2012 IEEE PES, vol., no., pp.1,8, 16-20 Jan. 2012

[10] Chenine, M.; Karam, E.; Nordstrom, L., "Modeling and simulation of wide area monitoring and control systems in IP-based networks," Power \& Energy Society General Meeting, 2009. PES '09. IEEE, vol., no., pp.1,8, 26-30 July 2009

[11] Marvin Rausand, Arnljot Hoyland, 'System Reliability Theory Models, Statistical Methods, and Application Second Edition', WILEY, 2004.

[12] Bertsch, J.; Carnal, C.; Karlson, D.; McDaniel, J.; Khoi Vu, "Wide-Area Protection and Power System Utilization," Proceedings of the IEEE, vol.93, no.5, pp.997,1003, May 2005 